\title{
Geometrical Multilayers: Coercivity in Magnetic 3D Nanostructures
}

\author{
P.A.J.de Groot ${ }^{\mathrm{a} *}$, A.A.Zhukov ${ }^{\mathrm{a}}$, R. Boardman ${ }^{\mathrm{b}}$, G.Bordignon ${ }^{\mathrm{b}}$, H.Fangohr ${ }^{\mathrm{b}}$, P.N.Bartlett ${ }^{\mathrm{c}}$ \\ ${ }^{a}$ School of Physics and Astronomy, University of Southampton, Southampton, SO17 1BJ, UK \\ ${ }^{b}$ School of Engineering Sciences, University of Southampton, Southampton, SO17 1BJ, UK \\ ${ }^{c}$ School of Chemistry, University of Southampton, Southampton, SO17 1BJ, UK
}

Elsevier use only: Received date here; revised date here; accepted date here

\begin{abstract}
Electrodeposition of magnetic metals through self-assembly templates from polystyrene spheres is used for fabrication of magnetic nanostructures with $3 \mathrm{D}$ architectures. These arrays demonstrate unusual properties including an oscillatory dependence of the coercive field on film thickness. Numerical simulations reveal that ratio between the array period and the hole diameter in anti-dot array is a crucial parameter giving rise to qualitatively distinct magnetization reversal regimes.
\end{abstract}

(C) 2008 Elsevier B.V. All rights reserved

PACS: 75.30.Gw; 75.40.Mg; 75.60.Ej

Keywords: magnetic nanostructures; self-assembly; coercivity; magnetic anisotropy

Self-assembly methods are emerging as viable, low cost fabrication techniques to prepare magnetic nanostructures [1, 2]. Electrodepostion of magnetic metals via templates from self-assembly of polystyrene spheres produces novel structures with 3D architecture [3]. The samples from this fabrication method show periodical patterning in the direction perpendicular to the film plane. A novel oscillatory behavior has been observed, which is a manifestation of a new class of magnetic materials: geometrical multilayer structures [4]. In this work we explore the origins of the periodical variations in the reversal processes.

The samples have been prepared using a fabrication procedure described in Ref. [2-4]. Magnetic measurements have been performed using a VSM at room temperature for magnetic fields applied parallel to the film plane. Magnetic structures were obtained from micromagnetic simulations using the OOMMF software pack [5].

In Fig. 1 we present the dependence of coercive field $B_{\mathrm{c}}$ on the film thickness, $t_{\mathrm{f}}$. The results show pronounced oscillations in $B_{\mathrm{c}}$, which reach a maximum when the top surface of the film is near the centre of a layer of close packed spherical voids. In contrast for complete spherical layers the coercive field approaches a minimum.
These observations suggest that the shape-induced magnetic anisotropy of the film varies periodically with $t_{\mathrm{f}}$. To explore this point we ran numerical simulations on a $2 \mathrm{D}$ antidot array with a hexagonal configuration and different ratios between array period $P$ and hole diameter $D$. The parameters

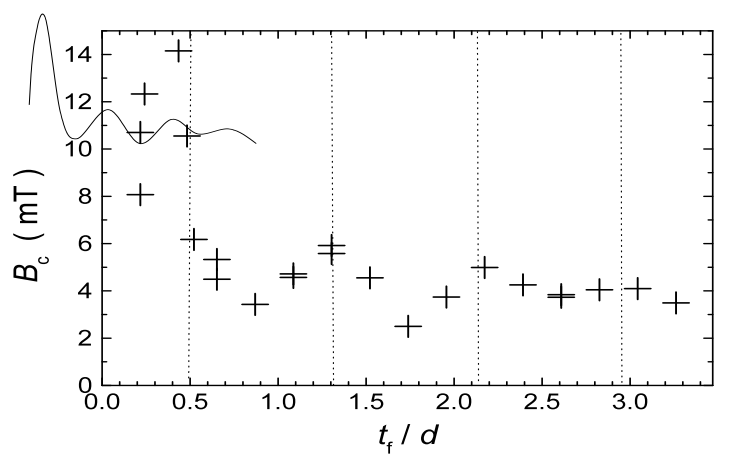

Fig. 1 Coercive fields for $\mathrm{Ni}_{50} \mathrm{Fe}_{50}$ films prepared from spheres with diameter $d=550 \mathrm{~nm}$ for different values of thickness, $t_{\mathrm{f}}$. The dashed lines indicate the positions of sphere centers for each layer in the close-packed structure.

* Corresponding author. Tel.: +44-23-80592110; fax: +44-23-80593910.

E-mail address: pajdeg@phys.soton.ac.uk. 


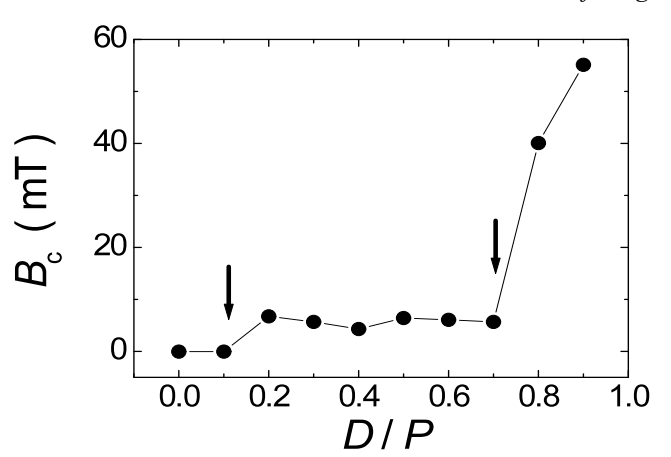

Fig. 2 Coercive field of films with hexagonal hole arrays from 2D numerical simulation as a function of the hole diameter $D$ for fixed period of $P=200 \mathrm{~nm}$.

for the micromagnetic modeling of $\mathrm{Ni}_{50} \mathrm{Fe}_{50}$ were chosen to be $A=5.85 \mathrm{pJ} / \mathrm{m} \quad$ (exchange), $\mu_{\mathrm{o}} M_{\mathrm{S}}=1.58 \mathrm{~T}$ (saturation magnetization) and $K=0$ (anisotropy). The thickness of the layer was taken equal to the cell lateral dimension of $3 \mathrm{~nm}$. As can be seen from Fig.2, $B_{\mathrm{c}}$ increases strongly when $D$ approaches $P$.

We have found similar behavior for different periods $P$ in the range of 50nm to 500nm [6]. For small diameters of holes we observe a sharp increase in the coercive field on increasing $D / P$ from zero. The critical point is roughly independent on $P$ and corresponds to $D_{\mathrm{c}} \approx 20 \mathrm{~nm}$. Detailed analysis of moment distribution reveals that this point corresponds to a transition from a parallel moment configuration (Fig.3a) to an onion state (Fig.3b) when magnetic moments align parallel to the edge of the hole to reduce the magnetostatic energy. We observe sharp switching for small diameters and for bigger diameters skewed curves reflecting the moment rotations. The critical size $D_{\mathrm{c}}$ for the first transition may be estimated from the balance between the exchange energy loss in the onion state $\sim 2 \pi A \ln (P / D) t_{\mathrm{f}}$ and magnetostatic energy of parallel state $\sim \mu_{\mathrm{o}} \pi^{2} t_{f}^{2} D M_{\mathrm{s}}^{2} / 2$ using the thickness of the layer $t_{\mathrm{f}}=3 \mathrm{~nm}$. In this simple estimate we neglect the onion core contribution. We then get $D_{\mathrm{c}} \approx 16 \mathrm{~nm}$ in good agreement with the simulations.

The second change in magnetization reversal happens when $D$ approaches $P$ resulting in a sharp increase in coercivity. This happens for $D / P=\sim 0.7$. At this point the direction of the onion core changes from being parallel (Fig.3b) to the base of the triangular structure to the $30^{\circ}$ direction (Fig.3b), which minimize the magnetostatic energy of onion core interaction.

This observation can be used to explain qualitatively

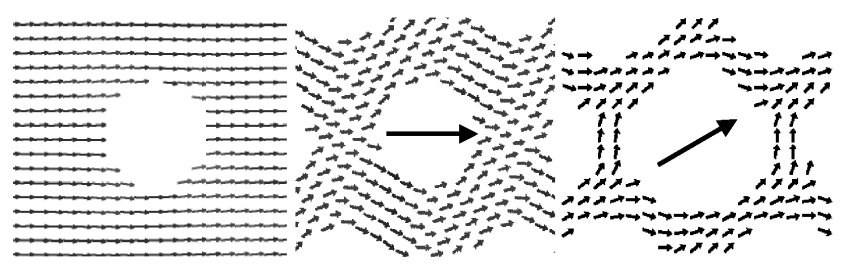
a)
b)
c)

Fig. 3 Moment distribution for $\mathrm{P}=100 \mathrm{~nm}$ and $\mathrm{D}=20 \mathrm{~nm}$ (a), $60 \mathrm{~nm}$ (b) and $80 \mathrm{~nm}$ (c). Arrows show the direction of the onion core.

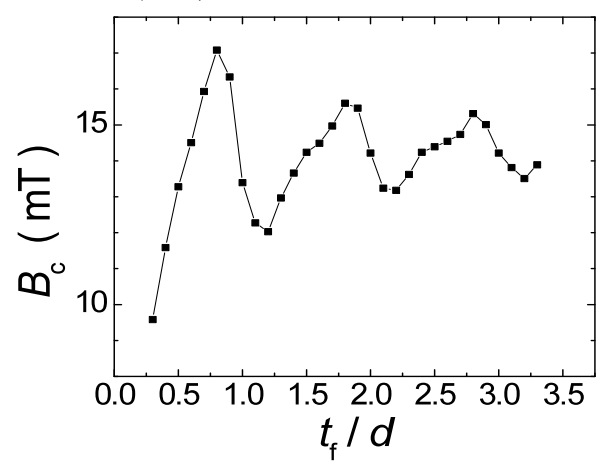

Fig.4 Calculated dependence of the coercive field from the homogeneous-layer micromagnetic model.

the experimentally observed oscillatory behavior, $B_{\mathrm{c}}\left(t_{\mathrm{f}}\right)$, in the $3 \mathrm{D}$ structure assuming that with variation of thickness we periodically add soft and hard magnetic layers depending on the $D / P$ ratio. Using the values of $B_{\mathrm{c}}(D / P)$ found from the $2 \mathrm{D}$ numerical simulations and taking $P=d$, we model structure as a 1D stack of layers with the anisotropy parameter for a given layer: $K(D / d)=m_{\mathrm{S}} B_{\mathrm{c}}(D / d) / 18$, with $m_{\mathrm{S}}$ the saturation magnetic moment of this layer. To avoid complications of a closepacked structure with overlapping spherical layers we simplify by choosing a simple cubic arrangement of spherical holes. Using a Monte-Carlo method we calculate $B_{\mathrm{c}}\left(t_{\mathrm{f}}\right)$ for the stack of exchange coupled layers. The results shown in Fig.4 reproduce qualitatively the experimental data. Note that the transverse period of the cubic structure is $d$ and differs from the value of $(2 / 3)^{1 / 2} d$ for the closed-packed configuration.

Using a bottom-up self-assembly fabrication method, we have prepared magnetic nanostructures with 3D ordered architectures. These arrays demonstrate oscillatory dependencies of the coercivity with variation of film thickness. This behavior is similar to that of alternating hardsoft multilayers. Based on our findings, we suggest that these 3D nanostructures represent a new class of magnetic system: geometrical multilayers. In these systems layering is due to different local geometries of the material rather than different compositions in the layers. 2D numerical simulations reveal that the nature of magnetization reversal of anti-dot arrays changes qualitatively with the size of holes. Using the 2D simulation results in a multilayer model reproduces the observed oscillatory coercivity behavior.

This work was supported by the Engineering and Physical Sciences Research Council (UK)

\section{References}

[1] J.Y. Cheng et al., Adv. Mater. 15 (2003) p.1599.

[2] P.N.Bartlett et al., J.Mater.Chem. 13 (2003) 2596.

[3] A.A.Zhukov et al., IEE Proc.-Sci.MeasTechnol., 150 (2003) 257.

[4] A.A.Zhukov et al., Appl.Phys.Lett., 88 (2006) 062511.

[5] The "Object Oriented Micromagnetic Framework" developed at NIST, Gaithersburg - http://math.nist.gov/oommf/.

[6] G.Bordignon et al., in preparation. 